Ministro de Salud y Protección Social

Alejandro Gaviria Uribe

\section{Directora General Instituto Nacional de Salud}

Martha Lucía Ospina Martínez

\section{Director de Vigilancia y Análisis del Riesgo en Salud Pública}

Máncel Enrique Martínez Durán

\section{Comité Editorial}

Oscar Eduardo Pacheco García

Hernán Quijada

Pablo Enrique Chaparro Narváez

Orlando Castillo

Vilma Fabiola Izquierdo

Alfonso Rafael Campo Carey

Natalia Muñoz Guerrero

Santiago Fadul

Máncel Martínez Ramos

Edición y corrección de estilo

Máncel Enrique Martínez Durán

\section{Diseño y Diagramación}

Claudia P. Clavijo A.

Dirección de Vigilancia y Análisis del Riesgo en Salud Pública, INS

\section{Instituto Nacional de Salud}

Avenida calle 26 n. ${ }^{\circ} 51-20$

Bogotá, D.C., Colombia

El Informe Quincenal Epidemiológico Nacional (IQEN) es una publicación de la Dirección de Epidemiología y Demografía del Ministerio de Salud y Protección Social y de la Dirección de Vigilancia y Análisis del Riesgo en Salud Pública del Instituto Nacional de Salud.

Los datos y análisis pueden estar sujetos a cambio. Las contribuciones enviadas por los autores son de su exclusiva responsabilidad, y todas deberán ceñirse a las normas y principios éticos nacionales e internacionales.

El comité editorial del IQEN agradece el envío de sus contribuciones a la Dirección de Vigilancia y Análisis del Riesgo en Salud Pública del Instituto Nacional de Salud; mayor información en el teléfono 22077 00, extensiones 1382, 1486.

Cualquier información contenida en el IQEN es de dominio público y puede ser citada o reproducida mencionando la fuente.

\section{Cita sugerida:}

Comportamiento epidemiológico de los casos de intoxicaciones por solventes

en Colombia, 2011 - 2015; 21 (12): 308 - 319. 


\title{
Brote de intoxicación aguda por exposición ocupacional a plaguicidas, La Calera, Cundinamarca, Colombia, 2016
}

\author{
María Nathalia Muñoz Guerrero' ${ }^{1}$ \\ Karla Mabel Cárdenas Lizarazo² \\ Diana Bustos ${ }^{3}$
}

Efrén Rojas ${ }^{4}$

\section{Resumen}

Antecedentes: El 18 de mayo de 2016 se notifica un brote de intoxicación aguda por plaguicidas, afectando cinco agricultores de un cultivo de papa (consumo de una bebida artesanal contaminada) y 24 trabajadores de la salud (exposición indirecta vía inhalatoria durante la atención de pacientes). Debido a la situación presentada el Instituto Nacional de Salud activó un equipo de respuesta inmediata para dar apoyo, con los objetivos de describir las características demográficas y sociales de la población afectada, identificar las características clínicas y epidemiológicas de los casos, además de determinar la frecuencia y distribución de los casos de intoxicación por plaguicidas en el municipio de La Calera, Cundinamarca.

Métodos: Se realizó un estudio de brote cuya población se conformó por los habitantes de la zona rural y el personal de urgencias de la Clínica que cumplía con las definiciones de caso. Se calcula proporciones de datos nominales y medidas de tendencia central, presentadas en tablas de frecuencia, calculando diferencias estadísticas e intervalo de confianza del 95\%.

Resultados: El número de casos del brote fue de 29 de los cuales el 75,9 \% fueron hombres, el 82,2 \% de los casos se presentó en el personal de salud que atendieron a los cinco agricultores afectados inicialmente, el 58,6 \% no requirieron hospitalización. La vía de exposición predominante fue la respiratoria. Durante la investigación de campo, se encontró la presencia de productos con ingredientes activos profenofos y acefato, los cuales son plaguicidas organofosforados. Según la revisión de las historias clínicas presentaron síntomas por intoxicación con un inhibidor de acetilcolinesterasa.

Conclusiones: Los casos con exposición directa e indirecta cumplen con la definición de caso de intoxicación por plaguicidas, por tanto se recomienda la adherencia de protocolos de bioseguridad en la atención de emergencias por sustancias químicas.

\section{Palabras clave:}

Intoxicación, plaguicidas, brote, ocupacional, inhibidores de colinesterasa, organofosforados, comunicación del riesgo.

\footnotetext{
1.Ingeniera Sanitaria y Ambiental, Esp. Epidemiología, MSc. Salud Pública, Epidemióloga de campo FETP. Grupo de Factores de Riesgo Ambiental. Dirección de Vigilancia y Análisis del Riesgo en Salud Pública. Instituto Nacional de Salud.

2 Médica, MSc. Toxicología. Grupo de Factores de Riesgo Ambiental. Dirección de Vigilancia y Análisis del Riesgo en Salud Pública. Instituto Nacional de Salud.

3 Bacterióloga Esp. Epidemióloga. Secretaria de Salud de Cundinamarca. Subdirección de Vigilancia en Salud Pública

4 Ingeniero químico Esp. Seguridad y prevención de riesgos profesionales. Esp. Evaluación de Impacto ambiental. Secretaria de Salud de

Cundinamarca. Subdirección de Vigilancia en Salud Pública
} 


\section{IQEN I WFOOME EUUMCENAL EPIDEMIOLÓGICO NACIONAL}

\section{Antecedentes}

El 18 de mayo de 2016 se reciben en la Dirección de Vigilancia y Análisis de Riesgo en Salud Pública del INS la notificación por la oficina de epidemiología de Cundinamarca, informando que en el municipio de La Calera, Sector Los Trinos, vereda El Hato San José, se presentó un brote de intoxicación por plaguicidas en un cultivo de papa, donde se vieron afectados cinco hombres empleados del cultivo quienes se encontraban realizando actividades de fumigación en horas de la mañana, que cerca del medio día suspenden labores para tomar "guarapo" (bebida artesanal de la región preparada por el cocinero del cultivo); luego de su ingesta presentan vértigo y desmayo, de acuerdo con las versiones dadas por el propietario del cultivo, quien ante este suceso en colaboración con el cocinero realizan el traslado de manera inmediata a la Clínica Nuestra Señora del Rosario ESIMED de La Calera, quien luego de dejarlos en la IPS se ausenta, sin dar ningún tipo de identificación de los pacientes ni información de los hechos.

Al llegar a la sala de urgencias de la Clínica Nuestra Señora del Rosario ESIMED, uno de los agricultores ingresa sin signos vitales, los otros cuatro con convulsiones tónico clónicas generalizadas, uno de ellos en paro respiratorio requiriendo maniobras de reanimación avanzadas (intubación orotraqueal, tratamiento de las convulsiones con benzodiacepinas y tratamiento específico con atropina).

Debido a la magnitud de la situación presentada se requirió el apoyo de gran parte de los profesionales de la salud que laboran en la Institución, los cuales se vieron expuestos de manera indirecta al producto, presentando náuseas, vómito, cefalea y mareo, tres de ellos con lipotimia; el total de funcionarios de la clínica afectados fueron 24 (entre personal asistencial y administrativo). De igual manera, se informa que dos policías que apoyaron el ingreso de los afectados también presentaron síntomas leves que remitieron espontáneamente. El personal de salud de la Clínica Nuestra Señora del Rosario ESIMED recibió atención inmediata con medidas de descontaminación, encontrándose estables al momento de la notificación; tres funcionarios fueron remitidos a diferentes clínicas de Bogotá, siendo dados de alta el 19 de mayo sin ninguna complicación.

Una vez estabilizados los pacientes y debido a su condición clínica se requirió apoyo del municipio de Sopó para remitirlos de forma primaria así: uno a la Fundación Santa Fe y los tres restantes al Hospital Santa Clara de Bogotá.

Ante la situación presentada y a la información captada por medios de comunicación, donde se reportan alertas sanitarias por afectación de fuentes hídricas que ponen en riesgo la salud de los habitantes de tres municipios de Cundinamarca (Sopó, Guasca y La Calera) (figura 1), el 19 de mayo a las 6 am se activa un equipo de respuesta inmediata del Instituto Nacional de Salud conformado por profesionales del Grupo de gestión de riesgo y respuesta inmediata y del Grupo de factores de riesgo ambiental, responsables de la vigilancia en salud pública de las intoxicaciones por sustancias químicas, realizando unidad de análisis para apoyar a la entidad territorial y al municipio en la atención del evento presentado. 


\section{IQEN

Figura 1. Noticias - Monitoreo de medios de situación de emergencia en el municipio La Calera, Colombia, 2016

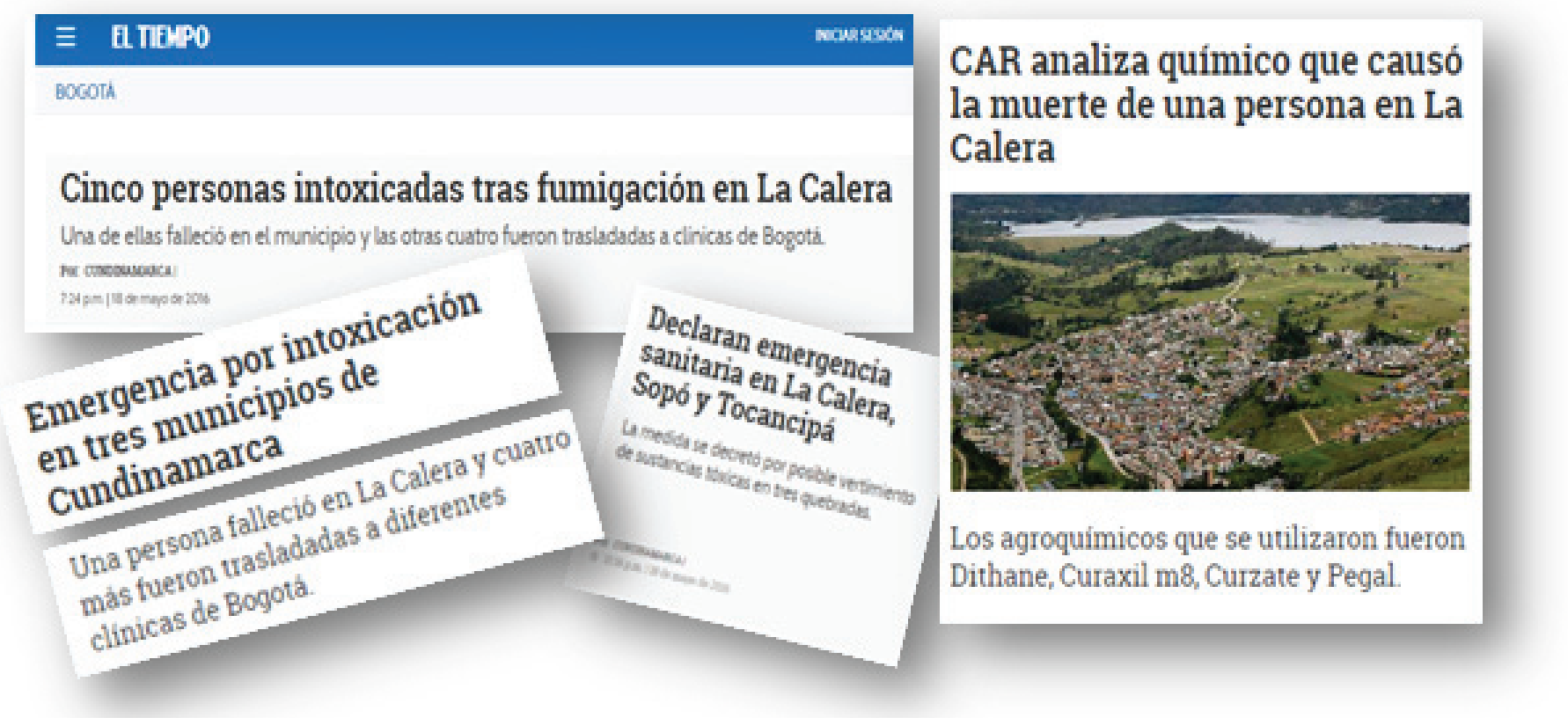

Fuente: Medios de comunicación 2016.

Teniendo en cuenta el impacto en la salud que ocasiona el uso de plaguicidas en los agricultores, debido principalmente a la toxicidad de estos productos y las prácticas inadecuadas en el manejo durante y después de su uso $(1,2)$, lo que genera efectos de manera inmediata (intoxicaciones agudas) y a largo plazo (intoxicaciones crónicas) (3); se realiza el estudio del brote de intoxicación con el fin de describir las características demográficas y sociales de la población afectada, identificar las características clínicas y epidemiológicas de los casos, además de determinar la frecuencia y distribución de los casos de intoxicación por plaguicidas en el municipio de La Calera, Cundinamarca.

La Calera es un municipio colombiano, ubicado en el occidente de la provincia del Guavio en el departamento de Cundinamarca. El municipio está ubicado al nororiente de Bogotá, cerca de la localidad de Chapinero.
La Calera se halla sobre la vereda Maniblanca, a orillas del río Teusacá. Se encuentra entre los 2600 y 3000 msnm por lo que tiene pisos térmicos frío y páramo. Su economía es variada ya que incluye la agricultura tradicional de papa, maíz, cubios, zanahorias etc; la ganadería de vacunos, caballos, ovejas, cabras, piscicultura de truchas etc. Por otro lado hay una importante explotación minera de piedra caliza, areneras y una industria que incluye la producción de cemento; además, dentro de la jurisdicción de La Calera está las dos grandes represas que surten al Acueducto de Bogotá San Rafaél y Chingaza. El turismo es también importante porque se puede acceder al Parque Nacional Chingaza, al mirador sobre Bogotá, a la zona de discotecas y muchas fincas de recreo quedan en La Calera. 


\section{IQEN INFOANE QUUNCENAL EPIDEMIOLÓGICO NACIONAL}

Mapa 1. Municipio La Calera, Área de ocurrencia del brote, Cundinamarca, Colombia, 2016

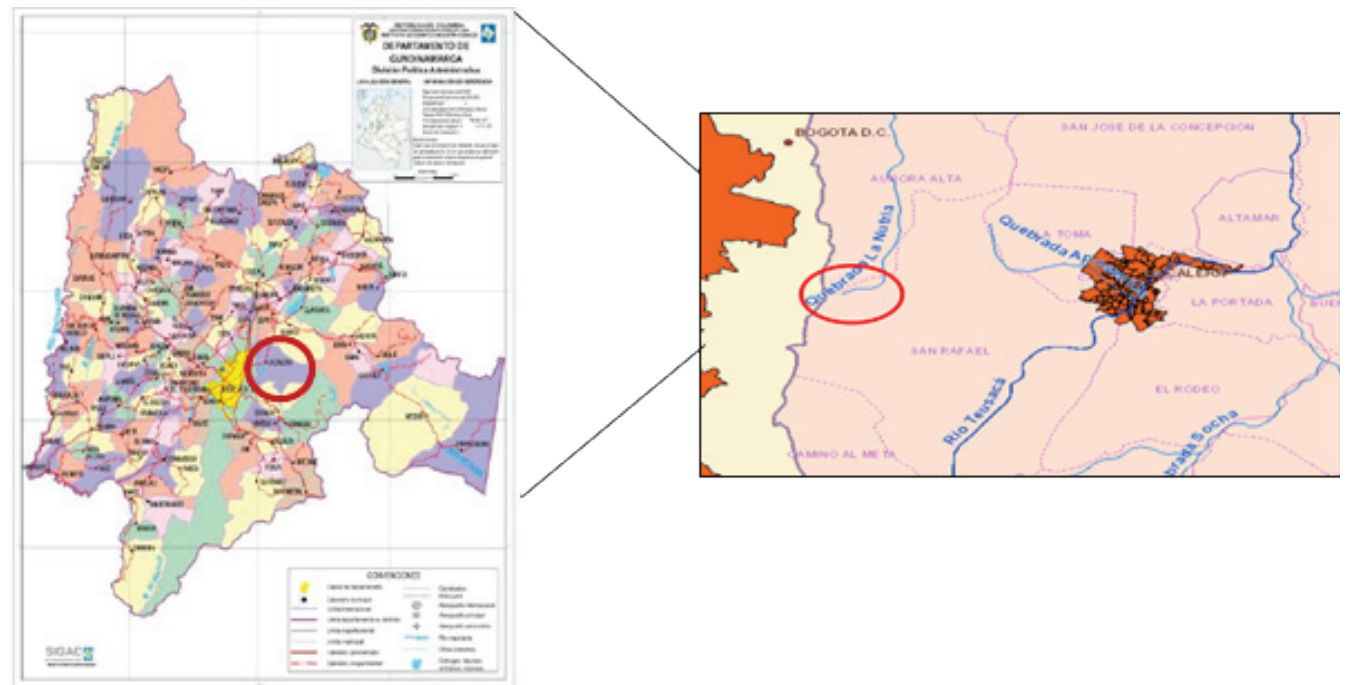

Fuente: http://mapas.cundinamarca.gov.co/flexviewers/20140808_Municipios/

En el departamento de Cundinamarca, durante el 2014 se notificó 1.173 casos individuales de intoxicaciones agudas por sustancias químicas, de las cuales 368 correspondían a intoxicaciones por plaguicidas mientras que para el mismo periodo del año 2015 se notificó 1.575 casos individuales, de los cuales 434 fueron por plaguicidas; para éste último año los productos mayormente reportados fueron "Campeón" (rodenticida de venta ilegal que no cuenta con registro sanitario) y carbamatos.

Para el municipio de La Calera la notificación por intoxicación aguda por plaguicidas en 2014 fue de tres casos y en 2015 de un caso, en este último el producto implicado fue Campeón (4).

\section{Métodos usados en terreno}

\subsection{Tipo de estudio}

Se realizó un estudio de brote.

\subsection{Población en estudio}

Habitantes del municipio de La Calera zona rural sector Los Trinos, vereda San Rafael y personal de urgencias de Clínica Nuestra Señora del Rosario ESIMED de La Calera.

\subsection{Variables}

Las variables en estudio fueron sexo, ocupación, área, afiliación al Sistema General de Seguridad Social en Salud, tipo y vía de exposición, síntomas, tratamiento utilizado, consulta al médico, hospitalización, nexo epidemiológico, medidas a nivel nominal; edad, número de casos, número de fallecidos, medidas a nivel de razón.

\subsection{Plan de recolección de datos}

Para la caracterización del brote del evento de interés en salud pública de Intoxicación aguda por plaguicidas, ocurrido en agricultores y profesionales de la salud del municipio, se realizaron las siguientes actividades:

Revisión de las actividades adelantadas hasta ese momento por la Secretaría de Salud Departamental de Cundinamarca; en este proceso se informa sobre el fallecimiento de un agricultor y varios afectados del personal de urgencias de Clínica Nuestra Señora del Rosario ESIMED de La Calera.

Revisión de las historias clínicas de los pacientes atendidos en la Clínica Nuestra Señora del Rosario ESIMED de La Calera, así como las historias clínicas de los pacientes remitidos al Hospital Santa Clara y a 
la Fundación Santa Fe en la ciudad de Bogotá, donde fueron atendidos por médicos toxicólogos. Dicha revisión se realizó por un equipo interdisciplinario que contaba con el apoyo de profesionales en epidemiología y toxicología, para su posterior diligenciamiento del formato único y estandarizado de unidades de análisis de casos especiales y muertes de eventos de interés en salud pública elaborado y diseñado por el Instituto Nacional de Salud.

Se obtuvo datos de los casos relacionados con el evento, notificados al evento de intoxicaciones por sustancias químicas (código 365) grupo plaguicidas del Sistema de Vigilancia en Salud Pública de Colombia (Sivigila) en la semana epidemiológica 20.

Se definió como caso:

- Persona trabajadora de cultivo de papa, ubicado en el municipio de La Calera, vereda San Rafael, sector Los trinos, quienes el 18 de mayo de 2016 se encontraban fumigando con plaguicida desconocido y que presentan sintomatología relacionada a intoxicación aguda por un inhibidor de colinesterasa.

- Personal de salud que se encontraba laborando en sala de urgencias de la Clínica Nuestra Señora del Rosario ESIMED de La Calera, el 18 de mayo de 2016 en turno de la mañana y que presentaron náuseas, vómito, mareo, cefalea y en algunos casos desmayo, posterior a la atención de personas afectadas por intoxicación aguda por un inhibidor de colinesterasa.

Formulación de hipótesis

1) Intoxicación aguda por consumo de un plaguicida inhibidor de colinesterasa, vehiculizado por una bebida alcohólica artesanal, contaminado durante actividad de fumigación de cultivo de papa.

2) Intoxicación aguda en trabajadores de la salud, por la inhalación de vapores y aerosoles generados en el momento de la atención a los pacientes involucrados en brote inicial de intoxicación aguda por sustancia química en el cultivo de papa.

\subsection{Plan de análisis}

Los datos obtenidos se organizaron en distribuciones de frecuencia que se presentan en tablas; a los datos numéricos se calculó medidas de tendencia central y de dispersión; los resultados principales se midieron como tasas de ataque y proporciones.

\section{Aspectos éticos}

Se realizó una investigación sin riesgos con base en la Resolución 08430 de 1993 del Ministerio de Salud, ya que es un estudio de brotes en donde se utilizó el registro de datos. Se garantizó la confidencialidad de los datos obtenidos.

\section{Resultados}

De acuerdo con el informe realizado por la Secretaría Departamental de Salud de Cundinarmarca y la notificación al Sivigila el total de casos implicados en el brote de intoxicación aguda por plaguicidas ocurrido en el municipio de La Calera en la semana epidemiológica 20 fueron 29 , de los cuales el 22 casos (75,9 \%) fueron hombres con una diferencia estadística en relación con las mujeres, el 24 casos $(82,2 \%)$ de los casos se presentó en el personal de salud que atendieron a los cinco agricultores afectados inicialmente.

Según el estado civil los solteros fue el grupo principalmente afectado con el 17 casos $(58,6 \%)$ y con un mismo porcentaje no requirieron hospitalización. El mayor número de casos se presentó en el centro poblado relacionados con los profesionales de salud afectados en la Clínica Nuestra Señora del Rosario ESIMED de La Calera, representando una diferencia estadística del 1,06 IC 95\% $(1,07-1,15)$ en comparación con el área rural; el 25 casos $(86,6 \%)$ pertenecen al régimen contributivo y uno de los casos no se encontraba asegurado a ningún régimen de seguridad social en salud. El mayor número de casos se presentaron en personas adultas de 35 a 39 años, seguido de los grupos de 25 a 34 años (ver tabla 1). 
Tabla No. 1 Descripción de las variables demográficas y sociales del brote de Intoxicación aguda por plaguicidas, La Calera, Cundinamarca, Colombia, 2016

\begin{tabular}{|c|c|c|c|c|c|}
\hline Variable & Categoría & Casos & $\%$ & $\mathbf{R R}$ & IC \\
\hline \multirow{2}{*}{ Sexo } & Mujer & 22 & 75,9 & 3,14 & $1,59-6,18$ \\
\hline & Hombre & 7 & 24,1 & & REF \\
\hline \multirow{2}{*}{ Ocupación } & Agricultores & 5 & 17,2 & & \\
\hline & Personal Salud & 24 & 82,8 & & \\
\hline \multirow{2}{*}{ Estado Civil } & Soltero & 17 & 58,6 & & \\
\hline & Casado & 12 & 41,4 & & \\
\hline \multirow{2}{*}{ Área } & Centro Poblado & 24 & 82,8 & 4,80 & $2,12-10,83$ \\
\hline & Rural Disperso & 5 & 17,2 & & REF \\
\hline \multirow{2}{*}{ Hospitalización } & $\mathrm{Si}$ & 12 & 41,4 & & \\
\hline & No & 17 & 58,6 & & \\
\hline \multirow{3}{*}{$\begin{array}{l}\text { Tipo de Régi- } \\
\text { men en Salud }\end{array}$} & Contributivo & 25 & 86,2 & & \\
\hline & No asegurado & 1 & 3,4 & & \\
\hline & Subsidiado & 3 & 10,3 & & \\
\hline \multirow{7}{*}{$\begin{array}{c}\text { Grupos de edad } \\
\text { (años) }\end{array}$} & 15 a 19 & 1 & 3,4 & & \\
\hline & 20 a 24 & 4 & 13,8 & & \\
\hline & 25 a 29 & 6 & 20,7 & & \\
\hline & 30 a 34 & 6 & 20,7 & & \\
\hline & 35 a 39 & 8 & 27,6 & & \\
\hline & 40 a 44 & 3 & 10,3 & & \\
\hline & 45 a 49 & 1 & 3,4 & & \\
\hline
\end{tabular}

La curva epidémica se elaboró con base en los datos de la hora de exposición a la sustancia, ya que no se cuenta con el dato exacto del inicio de síntomas; sin embargo estos se presentaron minutos después de la exposición de acuerdo con la información obtenido de la investigación inicial y de las historias clínicas analizadas; los primeros cinco casos corresponden a los agricultores presentando su exposición a las 11:30 am, seguido de los trabajadores de la Clinica del municipio, presentándose una tasa de ataque del 100\% (ver figura 2). 


\section{IQEN

Figura No. 2 Curva epidémica del brote de Intoxicación aguda por plaguicidas, La Calera, Cundinamarca, Colombia, 2016

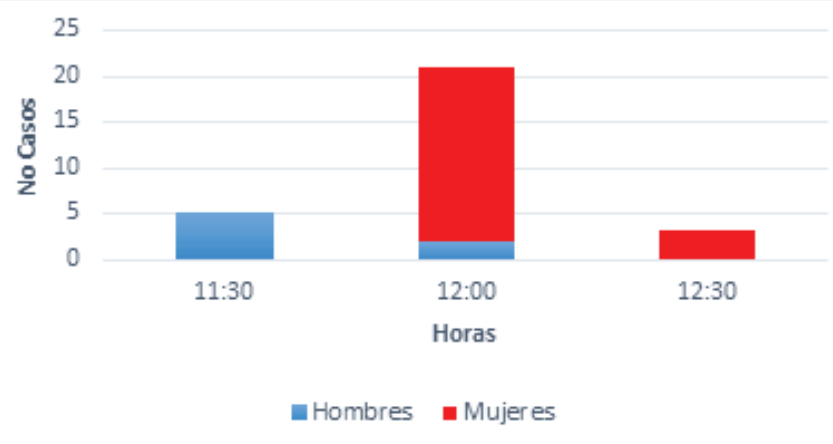

Fuente: Investigación epidemiológica de campo.

De acuerdo con las variables de exposición 24 casos (82,8 \%) se presentaron con vía de exposición respiratoria; estos casos están relacionados a los profesionales de la salud que atendieron a los agricultores los cuales presentaron la vía de exposición oral cinco casos (17,2 \%). En relación al tipo de escolaridad, 16 casos (55,2 $\%)$ se presentaron en técnicos profesionales, y profesionales; seis casos $(20,7 \%)$ en tecnólogos (ver tabla 2$)$.

Tabla 2. Descripción de las variables de exposición del brote de Intoxicación aguda por plaguicidas, La Calera, Cundinamarca, Colombia, 2016

\begin{tabular}{cccc}
\hline Variable & Categoría & Casos & \% \\
\hline \multirow{2}{*}{ Vía Exposición } & Respiratoria & 24 & 82,8 \\
& Oral & 5 & 17,2 \\
\hline Tipo de Exposición & Ocupacional & 29 & 100 \\
\hline \multirow{2}{*}{ Escolaridad } & Media Técnica & 1 & 3,4 \\
& Técnica Profesional & 8 & 27,6 \\
& Tecnológica & 6 & 20,7 \\
& Profesional & 8 & 27,6 \\
& Especialización & 1 & 3,4 \\
& Sin información & 5 & 17,2 \\
\hline Fuente: Investigación epidemiológica de campo.
\end{tabular}

De acuerdo con los hallazgos presentados en la Investigación epidemiológica de campo se tiene las siguientes consideraciones:

1- Por indagaciones hechas por la SIJIN de la Policía Nacional en el sitio de los hechos, se encontraron envases de plaguicidas presuntamente involucrados en la intoxicación con los siguientes nombres comerciales: Curzate M8 $\circledast$, Curaxil $®$ y Pegal PH $®$, éste último corresponde a un coadyuvante de uso agrícola.

2- El equipo técnico del INS, que apoyó en la investigación de campo participó en la reunión del comité local de emergencias, donde se hace énfasis en la reapertura de los servicios de urgencias y consulta externa de la Clínica del municipio y en acciones de comunicación del riesgo locales para la población de los tres municipios. 


\section{IQEN}

3- Posteriormente, los equipos del INS, de la Secretaría Departamental de Salud y del municipio se desplazaron a la vereda donde sucedieron los hechos con el fin de tomar muestras de agua de las fuentes hídricas que abastecen los tres municipios de la zona y a indagar los posibles nexos epidemiológicos de la población. Durante la investigación se identifica la mala disposición de envases vacíos de los plaguicidas, los cuales se encontraban esparcidos en toda la zona del cultivo y algunos usados en labores domésticas (ver foto 1).

Foto 1. Inadecuada disposición de envases de plaguicidas, brote de Intoxicación aguda por plaguicidas, La Calera, Cundinamarca, Colombia, 2016

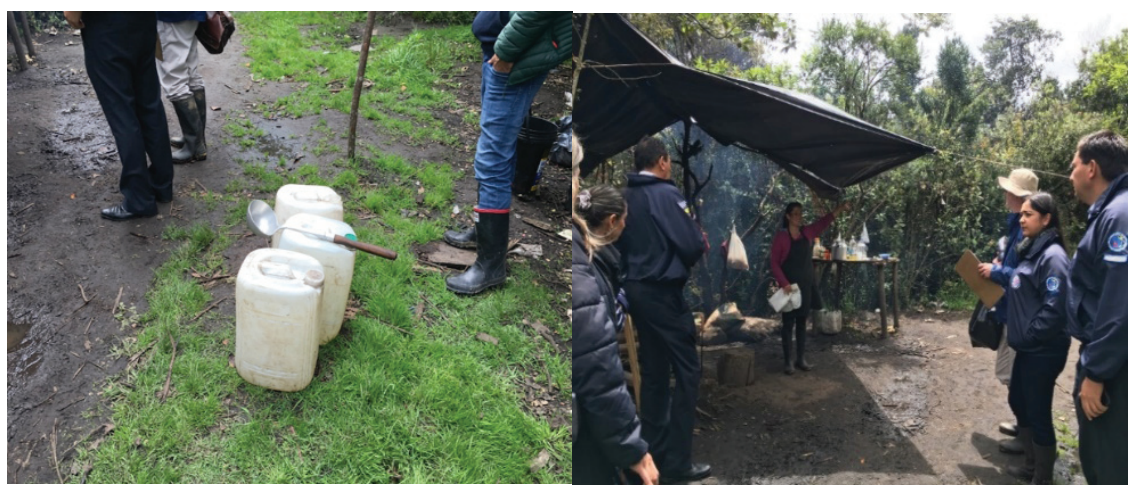

Fuente: Investigación epidemiológica de campo

4- La Secretaría de Salud de Cundinamarca inicialmente realiza una inspección preliminar del predio, posteriormente se inició la búsqueda de la fuente hídrica, denominada quebrada El Ajizal, para determinar los puntos en donde potencialmente se presentaría la mayor afectación. En el recorrido del terreno se encontraron canecas de 200 litros que habían sido utilizadas para mezcla de plaguicidas que aplicaron al cultivo. Es de anotar que dos de esas canecas se encontraban completamente llenas, tapadas con plásticos asegurados con ligas de caucho sin derrames aparentes a su alrededor. Estas canecas se encontraban ubicadas a menos de 20 metros de la ronda de la Quebrada Ajizal, fuente de abastecimiento de los Acueductos San José del triunfo y Pozo de la Nutria (ver foto 2 ).

Foto 2. Canecas con mezcla de plaguicidas usadas en el cultivo, brote de Intoxicación aguda por plaguicidas, La Calera, Cundinamarca, Colombia, 2016

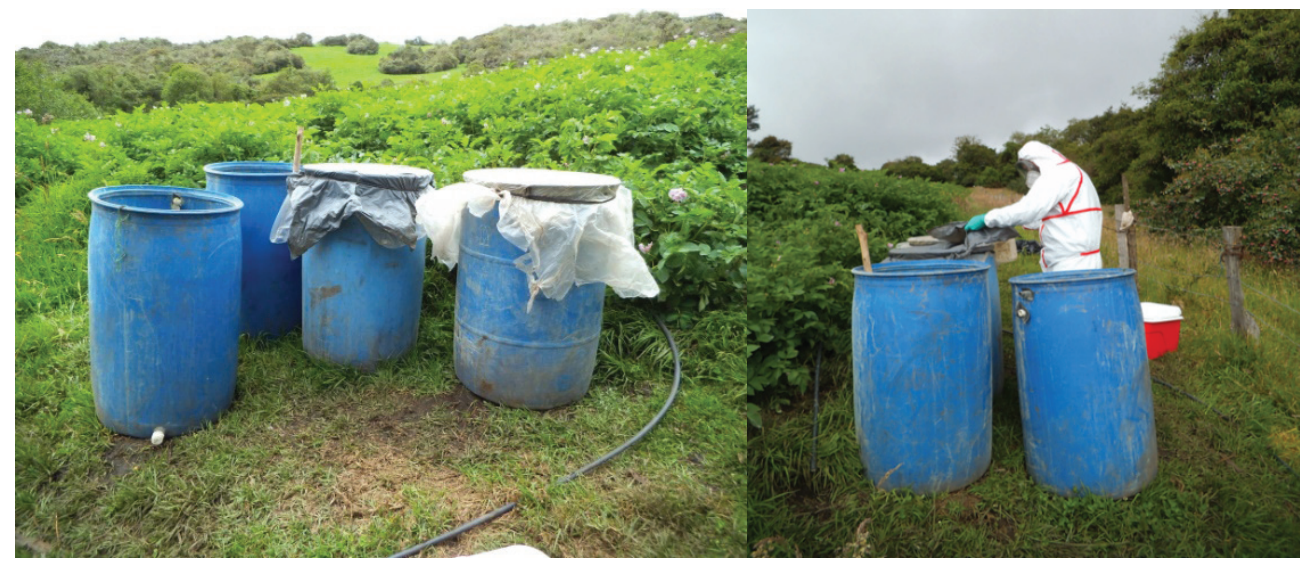

Fuente: Investigación epidemiológica de campo 


\section{IQEN

5- De igual manera, en contacto con funcionarios de la Defensa Civil y del Cuerpo Técnico de Investigación en la zona informan que obtuvieron muestras del "guarapo" consumido por los agricultores afectados, las cuales se encuentran en cadena de custodia y serán analizadas por el laboratorio de toxicología del Instituto Nacional de Medicina Legal y Ciencias Forenses.

6- Asimismo, durante la investigación de campo, se encontró la presencia de los productos Látigo y Magestic (con ingredientes activos profenofos y acefato, respectivamente), los cuales pertenecen al grupo químico de los plaguicidas organofosforados.

7- Se participa en el Consejo municipal de Gestión del Riesgo, donde se verifica una adecuada organización de los actores municipales; sin embargo, la comunicación del riesgo presenta falencias, lo que ha permitido la generación de rumores que han alertado a la comunidad.

8- Se cuenta con información sobre la mezcla de productos para la fumigación, mencionando el nombre y la dosis aplicada, sobre lo cual se realiza un análisis de acuerdo con los ingredientes activos de los productos y las dosis recomendadas en las etiquetas de los mismos (ver tabla 3 ).

Tabla 3. Descripción de mezcla utilizada Vs. dosis recomendada del brote de Intoxicación por Plaguicidas, La Calera, Cundinamarca, Colombia, 2016

\begin{tabular}{|c|c|c|c|}
\hline NOMBRE DEL PRODUCTO & PRINCIPIOS ACTIVOS & DOSIS APLICADA & DOSIS RECOMENDADA \\
\hline CURZATE M8 WP ( Categoría III ) & $\begin{array}{ll}\text { Mancozeb } & 640 \mathrm{~g} / \mathrm{Kg} \\
\text { Cimoxanil } & 80 \mathrm{~g} / \mathrm{Kg}\end{array}$ & $1000 \mathrm{~g} / 200 \mathrm{~L}$ agua & $500 \mathrm{~g} / 200 \mathrm{~L}$ agua \\
\hline DITHANE WP (Categoría III) & Mancozeb $800 \mathrm{~g} / \mathrm{Kg}$ & $500 \mathrm{~g} / 200 \mathrm{~L}$ agua & $600 \mathrm{~g} / 200 \mathrm{~L}$ agua \\
\hline FITORAZ WP 76 (Categoría III) & $\begin{array}{l}\text { Cymoxanil: } 60 \text { g/Kg } \\
\text { Propineb } 700 \text { g/Kg }\end{array}$ & $1000 \mathrm{~g} / 200 \mathrm{~L}$ agua & $670 \mathrm{~g} / 200 \mathrm{~L}$ agua \\
\hline LATIGO (Categoría IB) & $\begin{array}{l}\text { Clorpirifos } 600 \mathrm{~g} / \mathrm{L} \\
\text { Cipermetrina } 60 \mathrm{~g} / \mathrm{L}\end{array}$ & $200 \mathrm{ml} / 200 \mathrm{~L}$ agua & $250 \mathrm{ml} / 200 \mathrm{~L}$ agua \\
\hline CREOLINA & $\begin{array}{l}\text { Desinfectante fenólico } \\
\text { concentrado }\end{array}$ & $50 \mathrm{ml} / 200 \mathrm{~L}$ agua & $10 \mathrm{ml} / \mathrm{L}($ Uso veterinario) \\
\hline
\end{tabular}

9- Debido a la situación presentada y al desconocerse acerca de la disposición de los plaguicidas utilizados por los agricultores, en su momento la Corporación Autónoma Regional decidió cerrar de manera preventiva los acueductos que surten agua a tres municipios de Cundinamarca mientras se realizaron los respectivos análisis a las fuentes hídricas cuyos resultados fueron negativos.

Los cuatro casos remitidos a las instituciones de la ciudad de Bogotá cumplen con la definición de caso del evento de intoxicaciones por plaguicidas, más específicamente a un plaguicida inhibidor de la colinesteresa, teniendo en cuenta que posterior a la exposición a la sustancia se presentan manifestaciones clínicas compatibles con toxidrome muscarínico/nicotínico/sistema nervioso central, las cuales son de instauración rápida y responden al tratamiento específico (atropina). 


\section{IQEN}

Durante la investigación de campo se registra la presencia de los productos, Látigo y Magestic, que en su formulación tiene los ingredientes activos profenofos y acefato respectivamente, los cuales pertenecen al grupo químico de los plaguicidas organofosforados, grupo que se caracteriza por inhibir la enzima acetil colinesterasa, explicando la presentación de síntomas muscarínicos (miosis, sialorrea, aumento de secreciones pulmonares, disnea, vómito, bradicardia, entre otros), síntomas nicotínicos (fasciculaciones, hipertensión) y síntomas a nivel de sistema nervioso central (convulsiones, coma); considerando que la causa de intoxicación de los pacientes fue probablemente la exposición a alguno de estos productos, estando pendiente determinar si se trató de una exposición accidental durante sus labores agrícolas (ocupacional) o con algún tipo de intencionalidad.

Para el caso que terminó en desenlace fatal, éste cumple con la definición de caso del evento de intoxicaciones por plaguicidas, teniendo en cuenta que posterior a la exposición a la sustancia se presentaron manifestaciones clínicas compatibles con toxidrome muscarínico/nicotínico/sistema nervioso central las cuales fueron de instauración rápida, infortunadamente con desenlace fatal, el cual está relacionado con los otros cuatro casos de intoxicación que responden al tratamiento específico con atropina (nexo epidemiológico).

\section{Discusión}

Con la situación presentada se registra la falta de seguimiento de protocolos de bioseguridad el personal asistencial para la atención de emergencias con sustancias químicas, siendo necesario reforzar el adecuado uso de los elementos de protección personal en la atención de los pacientes intoxicados con éste tipo de productos por parte del personal de salud de las IPS públicas y privadas que operan a nivel municipal.

La falta de implementación de protocolos de comunicación del riesgo a nivel municipal fue determinante en la generación de rumores, desencadenando pánico en la comunidad, situación en la cual los medios ma- sivos de comunicación juegan un papel relevante, en especial aquellos transmitidos por televisión.

Teniendo en cuenta la situación presentada y de acuerdo con la información de los productos Curzate M8 $®$, Curaxil $®$ y Pegal PH $®$ (productos inicialmente implicados, según lo informado por el comité de riesgo del municipio de La Calera), se pudo establecer que estas sustancias tienen las siguientes características: las dos primeras son fungicidas con categoría toxicológica III y la tercera un coadyuvante con categoría toxicológica IV; en cuanto a los aspectos toxicológicos de las sustancias se encuentra que los ingredientes activos de los fungicidas son cimoxanil y mancozeb, de los cuales no existe soporte técnico y científico para pretender que éstas hayan sido la causa de una intoxicación aguda con los efectos presentados en las personas expuestas.

De igual manera, el diagnóstico de intoxicación por un plaguicida inhibidor de la colinesterasa no se explica por la exposición a las sustancias mencionadas anteriormente, para los cuales se esperaría como principales manifestaciones de intoxicación la presentación de síntomas inespecíficos como cefalea, mareo, debilidad y náuseas y en casos severos alteración del estado mental y convulsiones, sin las manifestaciones muscarínicas presentadas.

Sin embargo, durante la investigación de campo se encontraron envases de los productos Látigo y Magestic, en cuya composición se encuentra los ingredientes activos profenofos y acefato respectivamente, los cuales hacen parte del grupo de los organofosforados, grupo que se caracteriza por inhibir la enzima acetil colinesterasa con la presentación del toxidrome muscarínico/ nitcotínico/sistema nervioso central compatible con las manifestaciones clínicas presentadas principalmente por los agricultores afectados quienes presuntamente estuvieron expuestos a una mayor concentración del producto.

Se considera que el fallecimiento del agricultor posiblemente se debió a que estuvo expuesto a una mayor dosis de la sustancia, presentando una rápida instauración de los síntomas por inhibición de la enzima ace- 
til colinesterasa, con falla respiratoria por aumento de la producción de secreciones pulmonares posiblemente asociado a broncoespasmo, falleciendo previo su ingreso a la Clínica ESIMED. Esta situación va acorde con el comportamiento de las muertes que se presentan en países en vías de desarrollo, donde la mayor parte de las muertes ocasionadas por plaguicidas son el resultado de la exposición a Organofosforados y Carbamatos (5-7).

Para la situación presentada se pudo establecer que los productos Látigo y Magestic cuentan con categoría toxicológica II y III respectivamente, según los registros de venta del país; sin embargo, se estima que existe mínima información sobre la magnitud de los envenenamientos, así como del papel que juegan los diferentes tipos de plaguicidas, sin embargo se sabe que los organofosforados categoría toxicológica I y II causan unas 200.000 muertes / año (8).

En nuestro país, los plaguicidas inhibidores de colinesterasa (organofosforados y carbamatos) constituyen el grupo de insecticidas más frecuentemente utilizados en el control de plagas en los cultivos, programas de erradicación de vectores de importancia en salud pública y control de plagas en nivel domiciliario. También se utilizan otros grupos de plaguicidas como piretroides, fungicidas, herbicidas bipiridilos y fenoxiacéticos e inclusive organoclorados (actualmente prohibidos) $(9,10)$.

\section{Conclusiones}

- Los casos con exposición directa (agricultores) e indirecta (profesionales de la salud) cumplen con la definición de caso del evento de intoxicaciones por plaguicidas, específicamente un plaguicida inhibidor de la colinesterasa (presuntamente organofosforado de acuerdo con el hallazgo de los productos Látigo y Magestic), teniendo en cuenta que posterior a la exposición a la sustancia se presentan manifestaciones clínicas compatibles con toxidrome muscarínico/nicotínico/sistema nervioso central.
- Los municipios de Cundinamarca, en general no tienen la totalidad de equipos y entidades requeridas para enfrentar y contener este tipo de emergencias.

- Falta de seguimiento de los protocolos de bioseguridad en la atención de emergencias por sustancias químicas, tanto por la comunidad en general como en el personal asistencial.

- Los protocolos de comunicación del riesgo a nivel municipal no se encuentran debidamente implementados.

\section{Recomendaciones}

- Sensibilizar a la población y al personal médico en el uso adecuado de elementos de protección en el manejo de los plaguicidas.

- Capacitar al personal asistencial en los protocolos de bioseguridad y de salud pública ante una emergencia con sustancias químicas.

- Fomentar acciones intersectoriales con el objetivo de sensibilizar a la población frente a las señales de alerta en caso de presentarse intoxicación por esta sustancia, dada su alta capacidad de producir secuelas y muerte.

- Se hace necesaria la realización de convenios y alianzas que permitan la integralidad de las acciones para intervenir adecuadamente éste tipo de situaciones.

- Fortalecer las estrategias de prevención vigilancia y control (Metodología SARAR) en el adecuado uso y manejo de plaguicidas.

- Acciones de comunicación del riesgo al municipio con el fin de evitar una falsa alarma en la población. 


\section{IQEN

\section{Agradecimientos}

Expresamos nuestros más sinceros agradecimientos a las personas que apoyaron en la investigación epidemiológica de campo, así como a los doctores Mancel Enrique Martínez, Oscar Eduardo Pacheco, Hernan Quijada, Alfonso Campo del Instituto Nacional de Salud, a la doctora Tatiana Diaz Hernandez, Marisol López Martinez de la Secretaria de Salud Departamental de Cundinamarca, al Ing. Carlos Molina de la Secretaria de Salud Municipal de la Calera por sus valiosos aportes en la obtención de la información en trabajo de campo.

\section{Bibliografía}

1. Páez M, Varona M, Díaz S, Castro R, Barbosa E, Carvajal N y Londoño A. Evaluación de riesgo en humanos por plaguicidas en tomate cultivado con sistemas tradicional y BPA (Buenas Prácticas Agrícolas). Revista de Ciencias, 2011;15:153-66.

2. Varona M., Díaz S., Henao G., Lancheros A., Murcia A., Morato R., et al. Exposure Factors to Organophosphate and Carbamates Pesticides in the Putumayo Department, 2006. Libro: Pesticides, Capítulo: The Impacts of Pesticides Exposure, ISBN 978-953-307-531-0. Publicado por: InTech, enero 2011.

3. Ymelda Montoro, Rocío Moreno, Luis Gomero, Maria Reyes L et al. Características de uso de plaguicidas químicos y riesgos para la salud en agricultores de la sierra central del Perú. Rev. perú. med. exp. salud pública, 2009;26(4):466-72. ISSN 1726-4634.

4. Instituto Nacional de Salud. Informe epidemiologico de la slntoxicaciones por sustancias quimicas, 2015. Disponible en: http://www.ins.gov.co/lineas-de-accion/Subdireccion-Vigilancia/Informe\%20de\%20Evento\%20Epidemiolgico/INTOXICACIONES\%20Periodo\%20XIII\%202015.pdf

5. Cárdenas $\mathrm{O}$, Silva E, Ortiz J. Uso de inhibidores de acetilcolinesterasa en once instituciones de salud, Colombia, 2002-2005. Biomédica, 2010;30(1):95-106. ISSN 0120-4157.

6. Organización Mundial de la Salud. Cepis/OMS. Recuperado el 01 de Marzo de 2011, de Curso de Autodiagnóstico, tratamiento y Prevención de intoxicaciones agudas por Plaguicidas: http://cepis.ops-oms.org/ tutorial2/e/index.html

7. Jaga K, Dharmani C. Sources of exposure and public health implications of organophosphate pesticides. Pan Am J Public Health. 2003;14:171-85.

8. Clinical management of acute pesticide intoxication: prevention of suicidal behaviours. World Health Organization 2008. http://whqlibdoc.who.int/publications/2008/9789241597456_eng.pdf

9. Cardenas, O. Silva, E. Morales, L. Ortiz, J. Estudio epidemiológico de exposición aplaguicidas organofosforados y carbamatos en siete departamentos colombianos, $1998-2001$. Biomedica 2005; 25:170 - 80

10. Hidrovo A. Vigilancia de las Intoxicaciones con Plaguicidas en Colombia. Salud Pública2000;2(1):36-46 